3. The British immigrant who died from lung cancer at the earlier age, 45 to 54, emigrated from Britain at an average age of 30 years; those who died between 55 and 64 emigrated at an average age of 24 years.

4. The percentage of Union-born men who died of lung cancer and had temporarily resided in Britain was higher than the percentage of those dying of other diseases who had temporarily resided in Britain.

5. In South African rural areas the lung cancer mortality rate for men aged 45 to 64 years was very low for both non-smokers and moderate smokers, and increased only with heavy cigarette smoking. This suggests that, in the absence of atmospheric air pollution, smoking fewer than 20 cigarettes a day causes so little irritation to the bronchial cells that the risk of lung cancer is small. In both South Africa and the U.K. the elimination of air pollution from urban areas would substantially reduce the incidence of lung cancer even if no change occurred in smoking habits.

This research was made possible by the co-operation of Dr. H. M. Stoker, director of the South African Census
Bureau, and Mr. D. P. J. Botha, the South African Population Registrar, and their staff, who enabled me to trace and study the death certificates of the lung cancer patients and the controls. My wife undertook the massive correspondence of more than 6,000 letters and gave me her constant encouragement.

My thanks are also due to the hard work of my other assistants, Mrs. Borkum, Mrs. Munro, Mrs. Basford, Miss Jeffreys, Mrs. Hitge, and Mrs. Mitchell. Considerable advice was given me by Mr. G. F. Todd, of the Tobacco Manufacturers' Standing Committee of Great Britain. Market Research Africa permitted me to use the results of their recent smoking-habits survey.

The cost of this inquiry was met by a grant from the Tobacco Manufacturers' Standing Committee, London.

REFERENCES

Berkson, J. (1959). Proc. Mayo Clin., 34, 206.

Dean, G. (1959). Brit. med. J., 2, 852.

Doll, R., Hill, A. B., Gray, P. G., and Parr, E. A. (1959). Ibid., 1,322 .

Mills, C. A. (1960). Amer. J. med. Sci.. 239, 316.

Prindle, R. A. (1961). In The Air We Breathe, edited by S. M Farber 177 . Thomas, Springfield, Ill.

Stocks, P. (1958). A.R. Brit. Emp. Cancer Campgn, 1957, Suppl. to Pt. II.

\title{
CATARACT EXTRACTION AND DIABETES
}

\author{
BY \\ A. GORDON BECKETT,* B.M., M.R.C.P. \\ Consultant Physician, Willesden General and Acton Hospitals, London; late Senior Medical Registrar, \\ Royal Free Hospital, London \\ AND \\ H. E. HOBBS, F.R.C.S. \\ Ophthalmic Surgeon, Royal Free Hospital, London
}

For many years it has been recognized that diabetics are prone to cataracts. Indeed, Bence Jones (1865) wrote, "There is no special complication belonging to diabetes unless it be cataract." It was then recognized to occur " early, at 30 or even at 19." Bouchardat (1852), France (1859), and von Graefe (1860) all wrote on the higher incidence of cataract in diabetics. Some authorities recognized that the failure of vision was worse if albuminuria was also present (Bouchardat, 1852 ; Harley, 1865), but it is likely they did not realize that this was probably due to the presence of retinopathy, almost always associated with albuminuria.

True diabetic cataract is of characteristic morphology, usually occurring bilaterally at the same time, and more commonly in younger diabetics. These cataracts are associated with a period of uncontrolled severe diabetes and are sometimes present at the time of diagnosis, but may appear also after good diabetic control has been established. Very occasionally the lens changes are reversed with continued treatment of the diabetes (Neuberg et al., 1958) ; but surgical treatment is usually required. Much more common than diabetic cataract is the so-called "senile" cataract, which occurs at an earlier age in the diabetic. It is usually associated with vascular disease, poor control of the diabetes, and sometimes with a family history of cataract (Keen, 1960). Lens opacities may extend with varying degrees of speed, some remarkably slowly, but ultimately surgical extraction is required.

Extraction of the lens in the diabetic has been recognized for more than half a century to be a procedure fraught with risks greater than in the non-

*From January 1, consultant general physician, New End Hospital. diabetic. In pre-insulin days these risks were held to preclude operation in the large majority of cases. Thus Glegg (1920) reported that of the 1,660 cataract extractions performed at the Manchester Eye Infirmary between 1899 and 1920, only 87 were in diabetics and in a large proportion of these there was a serious postoperative complication.

After the introduction of insulin in 1922, treatment of the diabetic became much more satisfactory and operation a less hazardous procedure. Subsequent improvements in surgical technique contributed to this added safety, and the outlook for the diabetic with loss of vision due to cataract is much less gloomy. Nevertheless, in referring to this greatly altered outlook, Nutt (1953) was impressed with the small number of diabetics (30) presenting for operation in Sheffield during the five years which he reviewed, and Dollfuss et al. (1958) commented upon a similar conservatism at l'Hôpital de la Pitié in Paris. From our own study it seems likely that only a proportion of patients with visual loss primarily due to cataract have presented for operation, since the incidence of cataract in 900 patients with onset of diabetes after the age of 40 attending the Royal Free Hospital diabetic clinics is some $33 \%$, but only $27 \%$ of those with cataract have had lens extraction.

The complications feared in such operations are chiefly those of haemorrhage and infection. The possibility of haemorrhage, accompanied in exceptional cases by disastrous expulsion of the contents of the globe, provided in the past one of the main deterrents to surgery, and the frequent occurrence of an indolent hyphaemia, followed at times by secondary glaucoma, emphasized the operative risks from this cause. Delayed healing of the incision and severe post-operative 
infection leading to hypopyon and panophthalmitis have long been known to be greater hazards in diabetics. Iritis, without signs of frank infection, resistant to treatment, and also terminating in secondary glaucoma in some cases, further reduced the visual gains of cataract extraction in diabetics and occasionally necessitated enucleation. The more fluid vitreous of the eye of the diabetic, which more readily prolapses at operation, has been thought by some to predispose to such complications. With the frequency of such sequelae and the possibility that retinopathy, recognized or unsuspected, might prejudice the visual result, it is not surprising that the attitude to cataract extraction in diabetics has been one of extreme caution.

The effects of accurate diabetic control and of the adoption of modern operative techniques on this picture may be judged by the reduced incidence of complications and the improvement of the visual results in present-day practice. Thus Nutt (1953), contrasting the Sheffield series of 30 diabetic cataract extractions with 50 in nondiabetics, found no greater liability to sepsis or delayed wound healing: $80 \%$ of them achieved good or satisfactory vision. Dollfuss et al. (1958) found, in two separate groups of 60 and 34 cases of diabetic cataract extractions, an incidence of complications higher than that in non-diabetics; but good or satisfactory visual results were achieved in some $62.3 \%$. The series of 142 extractions in diabetics performed at University College Hospital which Greaves (1961) has reported produced a similar percentage (63) of patients with "useful vision."

A separate problem of the diabetic cataract is its possible association with diabetic retinopathy, which may contribute to the visual loss and add to the risks of operation. The two conditions commonly occur together, presumably because extended duration of the diabetes, its poor control, and the presence of associated vascular disease are aetiological factors common to both conditions. Indeed, of the patients with cataract attending the Royal Free Hospital diabetic clinics, referred to above, $41 \%$ also showed retinopathy of some degree. The presence of retinopathy must influence the final outcome, but how far it does so has not been adequately studied; Nutt's series included only four with retinopathy, while Dollfuss et al. (1958) did not refer specifically to the effect of this ocular manifestation of diabetes.

\section{Personal Experience}

Our own experience over the past 10 years confirms the belief that the results of cataract extraction in the diabetic are now much better and that the incidence of operative complications is greatly reduced, provided adequate precautions are taken. These include maximum control of the diabetes both before and after the operative period, topical antibiotic prophylaxis, accurate wound-suturing, and preferably intracapsular extraction of the lens. Suitably chosen general anaesthesia was used in most cases, and its benefits no doubt also contributed to the results.

The results of extraction of 69 cataracts in 45 diabetics aged 54 to 83 , with an average of 66.7 years, are reported here from observations ranging over periods of 3 months to 10 years, averaging 3 years. The technique employed was intracapsular in 58 and extracapsular in 11 cases. Details of the operative and post-operative complications and the vision achieved are tabulated separately.
Particular regard was paid to the presence and extent of diabetic retinopathy, its course after operation, and its effect upon the visual result. The presence of retinopathy was regarded simply as a bar to a perfect visual result, and, unless it was known to be gross, operation was considered for all cases in which the lens opacity appeared to be the major obstacle to vision. In a proportion of cases operation was undertaken chiefly to reduce the increasing visual disability-for example, when the probability of obtaining good reading vision was known to be precluded by macular damage.

Since the presence and severity of disease of the ocular vessels could be expected to affect both results and complications, cases have been classified into three groups: (1) those with no retinopathy either pre- or post-operatively; (2) those in whom retinopathy was observed first after operation; and (3) those in whom it was known to be present at the time of operation or could be suspected because of its presence in the fellow eye. In eight patients in group 3 (indicated separately in Table II) retinopathy was already present when the diagnosis of diabetes was first made. In general the severity of retinal changes in group 3 cases was greater than that in group 2 cases.

\section{Visual Results}

The visual results and complications are summarized in Table $\mathrm{I}$. The results of a contemporary series of 135 extractions of senile cataracts performed in similar circumstances by the same surgeon (H. E. H.) are also given for comparison. The average age of the latter

\begin{tabular}{|c|c|c|c|c|}
\hline Group & Good & Satisfactory & Poor & $\begin{array}{l}\text { Incidence of } \\
\text { Complications }\end{array}$ \\
\hline $\begin{array}{l}\text { Diabetic series : } \\
\text { 1. Free from retinopathy } \\
\text { 2. Retinopathy observed } \\
\text { after operation } . . \\
\text { 3. Pre-operative retino- } \\
\text { pathy : } \\
\text { Developing after diag- } \\
\text { nosis a } \ldots \\
\text { Present at diagnosis .. } \\
\text { Non-diabetic series }\end{array}$ & $\begin{array}{l}28 \% \\
8.3 \% \\
78 \cdot 6 \%\end{array}$ & $\begin{array}{l}10 \% \\
15 \cdot 5 \%\end{array}$ & $\begin{array}{l}50 \% \\
75 \% \\
8 \cdot 1 \%\end{array}$ & $\begin{array}{l}50 \% \\
66.7 \% \\
30.4 \%\end{array}$ \\
\hline
\end{tabular}

patients was 71.1 years, the range being from 41 to 92 years. The mean follow-up period in this series was two and a half years. As in the diabetic series, the visual results were influenced by the presence of known and unsuspected retinal lesions, responsible for poor results in six instances.

Visual acuities of $6 / 12$ to $6 / 6$ were classified as good, those of $6 / 36$ to $6 / 18$ as satisfactory, and those of less than $6 / 36$ as poor. The majority of patients in whom poor results were obtained had, nevertheless, sufficient improvement of vision to enable them to continue with some sighted occupation, so that the number certified as blind was seven. A poor result is therefore not necessarily synonymous with surgical failure.

Of the 69 eyes submitted to operation a good result was obtained in $48 \%$, a satisfactory one in $16 \%$, and a poor one in $36 \%$. Four of the poor results were due to post-operative complications, three to lesions unrelated to either diabetes or operation, and 18 to involvement of the macula in diabetic retinopathy. Of the seven patients who had a poor result in one eye, four achieved a satisfactory and three a good result in the other eye, and of the seven patients certified as blind the diabetic condition could in itself be held responsible in only four. 
As might be expected, group 1 has the highest proportion of good results $(80 \%)$, a figure comparable to that seen in the non-diabetic group. Among those with retinopathy it is gratifying to find that so large a proportion achieved and retained, in a number of cases for several years, good or adequate vision.

Analysis of cases which failed to achieve a good result (Table II) shows that in the great majority this failure resulted from the presence of retinopathy which involved the macula. In four cases ( $22 \mathrm{~L}, 24,36$, and 44) it has been seen as the result of remote complications

TABle II.-Analysis of Cases Which Failed to Achieve a Good

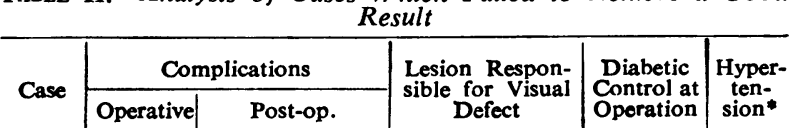

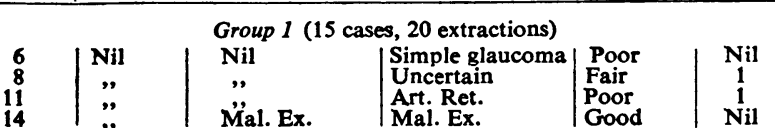

$$
\text { Group } 2 \text { (11 cases, } 19 \text { extractions) }
$$

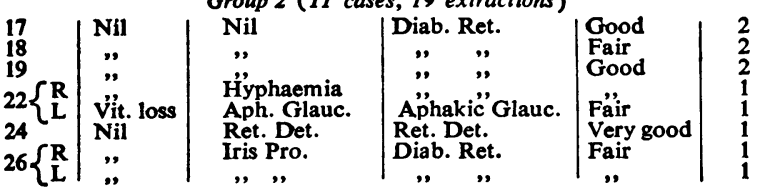

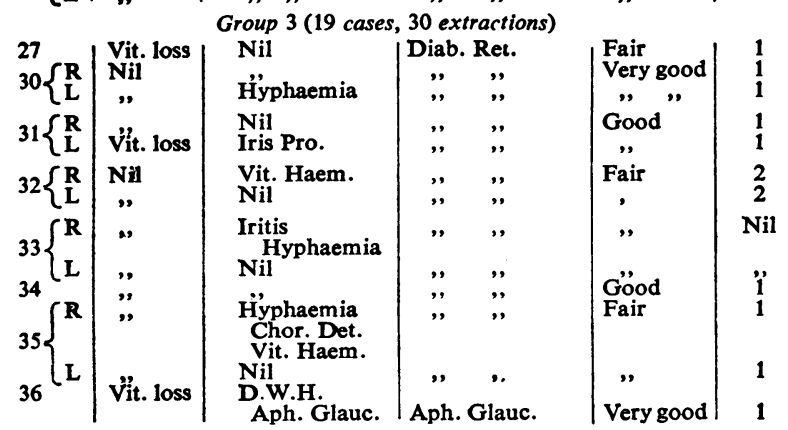

\section{Retinopathy Present at Diagnosis of Diabetes}

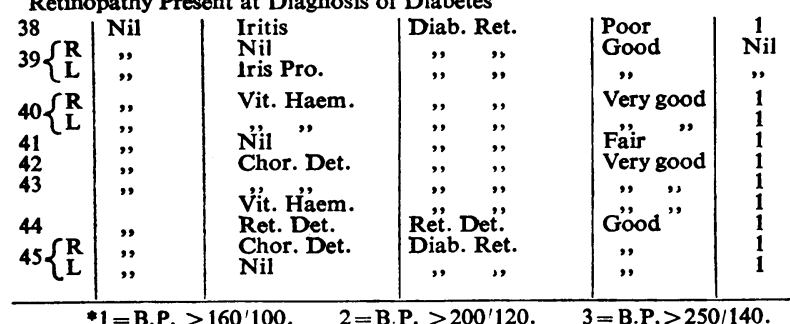

Mal. $1=$ B.P. $>160100 . \quad 2=$ B.P. $>200120 . \quad 3=$ B.P. $>250 / 140$ Ret. = Diabetic retinopathy. Aph. Glauc. = Aphakic glaucoma. Ret. Det. = Retinal detachment. Iris Pro. = Iris prolapse. Vit. Haem. = Vitreous haemorrhage. Chor. Det. = Choroidal detachment. D.W.H. = Delayed wound-healing. Art. Ret. = Arteriosclerotic retinopathy.

which are by no means peculiar to diabetes. In a further three (Cases 6, 11, and 14) it arose from the incidental presence of a non-diabetic condition. In only one case could poor diabetic control be held responsible for the poor visual result ; but the role of hypertension, noted with increasing frequency in groups 2 and 3 , is probably more important and is discussed below.

\section{Complications}

The total number of complications in the diabetic series was 40 , which occurred in 28 of 69 extractions, and in the non-diabetic series 41 , which occurred in 38 of 135 extractions (see Table III). In most cases, however (all instances of hyphaemia, iris prolapse, iritis, and choroidal detachment, and all but two of vitreous loss), these complications had no effect upon the ultimate visual result. The general incidence is comparable with the figures reported by Dollfuss et al. (1958), 55 of 94 extractions, and by Nutt (1953), 12 of 30 extractions. Individual complications show an expected variation in incidence and effect in the four

TABLE III.-Operative and Non-operative Complications

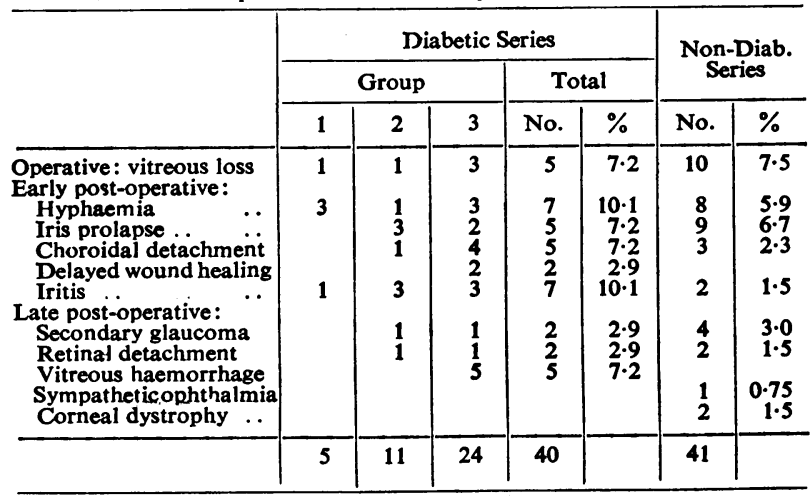

series. In none of them, however, has operative haemorrhage or post-operative hyphaemia been a serious problem. Post-operative iritis was seen with much the same frequency in all four, but responded better to treatment in our own (where no ultimate ill effects upon vision was observed) and in Greaves's (1961) series than in the French one (blindness in 3 of 12 affected eyes) or in that of Nutt (4 of 8 affected eyes enucleated). The proportion developing secondary glaucoma in our series $(2.9 \%)$ was lower than in the French series $(10.6 \%)$ and its effects upon vision were much less serious. This may perhaps be associated with the poorer response to treatment of iritis in that series.

Delayed wound-healing occurred in two of our cases. It was seen once by Nutt and eight times in Greaves's series, but did not occur in the French series, and is evidently not now as serious a hazard as formerly. In the French series, however, there was serious loss of vision in three cases as the result of a form of trophic keratitis which did not affect any of our cases.

The incidence of complications is seen (Table III) to be greater as the severity of the retinopathy increases. In the group of eight patients in whom this was manifest at the time of diagnosis (see above) it was at its highest, 8 of the 12 eyes in which extractions were performed being affected. These provided a large proportion of the complications seen as well as of the "poor" results of the whole series.

Intractable iritis and secondary glaucoma may still constitute risks which are greater in the diabetic than the non-diabetic eye; but the satisfactory response to treatment of the seven cases of post-operative iritis seen in the present series and the associated lower incidence of secondary glaucoma suggest that these risks need not rank as serious contraindications to operation.

\section{Diabetic Retinopathy and Operation}

Diabetic retinopathy is complex, with many different types of lesion: micro-aneurysms, venous distension, loops, and varicosities; haemorrhages; exudates; changes presumed to be the result of retinal ischaemia, to be followed by formation of " new" vessels and of fibrous tissue extending into the vitreous. The prognosis of retinopathy is uncertain. Although since the days of Mackenzie and Nettleship (1877) it has been recognized 
that occasionally certain of these lesions may remain static or regress, it is generally believed that its course is steadily downhill.

The cause of retinopathy and of its fluctuations is not known. Its incidence increases with the duration of the diabetes ; but it is becoming increasingly accepted that poor diabetic control, with its consequent metabolic effects, plays an important if not a crucial part. Thus all the 79 diabetics free from complications after 25 years of diabetes, and so awarded the "Joslin Victory Medal," were meticulously controlled over the whole period (Root et al., 1959). In many reports, such as those of Keiding et al. (1952), Dunlop (1954), White (1956a, 1956b), and Johnsson (1960), the incidence of retinopathy is highest in those with poor control of the diabetes.

In the diabetic with onset of disease in middle or later life, retinopathy is commonly present when the diabetes is recognized, the result of the insidious nature of the disease and the almost universal delay in diagnosis. In this group of diabetics treatment can be expected only to slow down or arrest the course of retinopathy and presumably cannot repair the damage already done to the retinal vessels. Such patients may suffer the consequences of major haemorrhages as the result of pre-existing damage, in spite of adequate therapy of their diabetes.

The effects of the trauma of operation upon such diseased vasculature and, conversely, the influence of its presence on operative complications and visual results are uncertain, but some light is thrown on these problems by the experience gained in the present study. The influence of retinopathy on the visual results is apparent from Table I. Where no retinopathy was detected good or satisfactory visual results were achieved in $90 \%$ of cases, whereas when retinopathy was detected only postoperatively the percentage fell to 73.5 , and where it had existed pre-operatively, often for long periods, to 40 . These differences are the result of the visual effects of retinitis proliferans or haemorrhage involving the macula.

This prejudicial effect of manifest vascular disease of the eye is seen even more clearly from the results in the small group of eight patients who had retinopathy at the time of diagnosis of their diabetes. In $75 \%$ of their eyes the visual result was poor and in only $16.7 \%$ was it satisfactory.

In a significant number of patients the retinopathy was observed to become haemorrhagic in the weeks or months following operation. In some this could be attributed to inadequate control, but in others this explanation could not be offered, and it seems reasonable to consider whether the changes occurring after operation could be held responsible, in particular as the result of the ocular hypotony produced. In respect of the major surgical haemorrhagic complications, as has already been demonstrated, the part which this plays in cataract extraction in the diabetic is no greater than that in the non-diabetic. Vitreous haemorrhage, which might properly be used as an index of the retinal vascular response to hypotony, resembles these in that it has been infrequent, occurring in only five eyes, only one of them in the early post-operative weeks. It is important to note, however, that one of these patients showed a greatly increased capillary fragility shortly before operation and that another, whose retinopathy progressed after operation, had increased fragility afterwards. Some with normal fragility suffered no such deterioration, but these were chance and isolated observations, not part of a planned investigation.

Also of interest is the case of a patient whose retinopathy became haemorrhagic and more extensive in the recently operated eye while that in the fellow eye, operated upon several years previously, remained slight and static. In another patient similar deterioration in retinopathy was seen in the operated eye but not in the unoperated one; this regressed after a year and is now minimal.

Hypertension of a moderate degree, found in the majority of elderly diabetics, especially those with retinopathy, may play some part in the deterioration of diabetic retinopathy. Although it is difficult to be certain of its precise role, almost all of our patients with a poor visual result due to an extension of retinopathy after lens extraction had some degree of hypertension. It seems reasonable to suggest that, in some patients at least, hypertension plays a part in causing retinal haemorrhage after the intraocular tension has been reduced by operation. Suitable pre-operative control of blood-pressure is evidently desirable, and the use of a hypotensive adjunct to anaesthesia, such as the pethidine and chlorpromazine mixture used in most cases in this series, may be advantageous.

\section{Discussion}

In spite of the great improvement in the treatment of diabetes brought about by insulin, the diabetic eye cannot be regarded as a normal one, as Nutt pointed out, when cataract extraction must be considered. The results of the present survey, however, justify the belief that the additional risks are much less severe than past reports suggest and that, when impaired vision is added to the other handicaps to which the diabetic is prone, operation can be considered in most cases with confidence that some degree of useful vision will be restored.

Among the factors responsible for the reduced incidence and severity of complications in this series, a prominent place must be given to improvements in surgical technique, which have now become standard practice. One of these is intracapsular extraction of the lens, as emphasized by Nutt, probably because of the smaller degree of intraocular irritation resulting from its complete removal. Also beneficial is the pressure upon the iris by the small air bubble injected into the anterior chamber at the conclusion of the operation, in that this may discourage haemorrhage from the diseased vessels of the iris and thus may contribute to the lower incidence of hyphaemia. Accurate suturing of the wound, technically possible only in recent years, must be largely responsible for the reduction in the incidence of delayed healing. Antibiotic prophylaxis and the use of steroids topically are undoubtedly important new aspects in the treatment of iritis.

Nevertheless the most important single factor is probably control of the diabetes. Direct evidence of the role of this control in reducing the incidence of operative and post-operative complications is not at once apparent from the results which we have quoted ; but their lessened severity is felt to be largely due to the degree of stability possible before, during, and after operation-chiefly the result of patient attention by conscientious house-surgeons and registrars. No patient was operated on if his diabetes was badly out of control. 
with dehydration or ketosis. More that half of the operations (42) were performed with either good or very good control ; but in eight cases, after prolonged attempts at stabilization, control remained poor. The influence of this control on the incidence of complications is uncertain in view of the small number seen ; but it is noteworthy that half of the cases of hyphaemia and four out of seven cases of iritis arose in patients in whom good control could not be achieved.

In the presence of retinopathy the possibility must be considered that it may extend post-operatively. Although this was believed to have happened in a few cases in this series, it is clearly not an inevitable consequence of operation. The possible role of hypertension in this respect has already been discussed. Another possible factor in this connexion is the increased capillary fragility of some diabetics. This was first described by Hanum (1939), particularly in association with retinopathy, and his findings were later confirmed (Foxworthy, 1945 ; Friedenwald, 1949 ; Barnes, 1950). Although Hanum noted its variability, no adequate study has yet been made, so far as we are aware, of the relationship between changes in capillary fragility and the various phases of retinopathy. The cases of vitreous haemorrhage associated with increased fragility in this series already referred to, as well as the general principles involved, suggest that some correlation may be found between increases in the haemorrhagic type of retinopathy (the form most damaging to vision after operation) and increased capillary fragility as demonstrated by the sphygmomanometer test. Friedenwald (1950) indeed suggests as much. It may well be that if an increased liability to post-operative progress of the retinopathy could be detected by means of the capillary fragility tests, then such tests might be used to indicate the most favourable time for operation. This particular aspect is being studied at the present time.

The cause of this increased capillary fragility in certain diabetics is not known. Early work by Friedenwald suggested that this might be deficiency of vitamin $C$, a theory which may account for Nutt giving large doses of vitamin $C$ to his patients before cataract extractions. Friedenwald (1949), however, subsequently reported that he could find no evidence of deficiency of ascorbic acid in diabetics, and no relationship of this to the abnormal capillary fragility.

With reduced operative risks, the indications for cataract extraction in the diabetic must be reconsidered. Operation can now be undertaken on purely visual grounds rather than late in the course of the cataract and the diabetes. On technical grounds this is desirable, as it facilitates the use of the intracapsular technique. Removal of the second cataract-an important factor in restoring mobility to aged patients-may be undertaken with greater confidence, and often confers greater benefit in the diabetic than in the non-diabetic, particularly if the result of the first operation has been poor as the result of chance involvement of the macula. This was, in fact, so in 7 of the 22 cases of the present series in which both cataracts were extracted, the result in the second eye being superior to that in the first.

Early operation is probably desirable also on general grounds. since the effects of hypertension and occlusive vascular disease, as well as the more specific diabetic disease of the ocular vessels, are likely to increase with the increasing duration of the diabetes; and with them the hazards of operation.

\section{Summary}

The indications for cataract extraction in the diabetic, its complications, and the operative and visual effects of retinopathy are reviewed in the light of a personal series of 69 such extractions. The operative results are compared with a similar personal series of 135 extractions in non-diabetics.

The incidence and severity of operative complications are found to be greatly reduced with present-day techniques when adequate control of the diabetes can be achieved: delayed wound-healing, sepsis, and secondary glaucoma are seen with but little greater frequency than in non-diabetics.

Ocular vascular disease, indicated by the presence of retinopathy, clearly provides the chief predisposition to haemorrhagic complications; but these, when they occur, are no longer disastrous, and in most cases are without prejudicial effect upon the ultimate visual result. Retinopathy does not invariably increase after operation, and may regress. If the macula is involved in it vision suffers; but it need not of itself be held to contraindicate operation and thus involve the acceptance of inevitable blindness. In view of the fact that the vascular disease tends to increase in severity as the disease continues, a particular indication for operating early rather than late arises in diabetics.

We are grateful to the physicians of the Royal Free Hospital whose cases have been confided to our care, and particularly to Dr. Una Ledingham, from whose diabetic clinics the majority of them were drawn.

\section{REFERENCES}

Barnes, R. H. (1950). Amer. J. med. Sci., 219, 368.

Bence Jones, H. (1865). Med. Tms Gaz., 1, 81.

Bouchardat, A. (1852). Du diabète sucré ou glycosurie, son traitement hygiénique. Baillière, Paris.

Dollfuss, M. A., Haye, C., and Penchon, S. (1958). Ann. Oculist (Paris), 191. 209.

Dunlop, D. M (1954). Brit. med. J., 2, 383.

Foxworthy, L. R. (1945). Cited by H. P. Wagener, Proc. Amer. Diab. Ass., 1945, 5, 203.

France (1859). Ophthal. Hosp. Rep., 1, 273.

Friedenwald, J. S. (1949). Amer. J. Ophthal., 32, 487.

(1950). Ibid., 33, I187.

Glegg, J. G. (1920). Trans. Ophthal. Soc. U.K., 40, 37

von Graefe, A. (1860). Jb. Fortschr. ges. Med., 4, 257.

Greaves, D. P. (1961). Proceedings of 3rd International Course in Ophthalmology. Barcelona.

Hanum, S. (1939). Acta ophthal. (Kbh.), 16, Suppl., p. 3.

Harley, G. (1865). Med. Tms Gaz., 2, 516.

Johnsson, S. (1960). Diabetes, 9, 1 .

Keen, H. (1960). Reported to the Scientific Section of the British Diabetic Association at Guy's Hospital, London.

Keiding, N. R., Root, H. F., and Marble, A. (1952). J. Amer. med. Ass., $150,964$.

Mackenzie, S., and Nettleship, E. (1877). Ophthal. Hosp. Rep., 9. 134.

Neuberg, H. W., Griscom, J. H., and Burns, R. P. (1958). Diabetes, 7, 21 .

Nutt, A. B. (1953). Brit. J. Ophthal., 36, 725

Root, H. F., Mirsky, S., and Ditzel, J.' (1959). J. Amer. med. Ass., 169, 903.

White, P (1956a) Diabetes, 4, 290.

- (1956b). Ibid., 5, 445 .

The Physiotherapist and the Remedial Gymnast, a revised booklet in the "Choice of Careers" series, has been published by the Central Youth Employment Executive. (H.M.S.O., price 1s. net.) The booklet deals with the work and training of physiotherapists and remedial gymnasts in the National Health Service, where the majority are employed, and also indicates other spheres of the profession, including the fighting services. Information is given about the educational qualifications required, the training courses available, openings and salaries, and there is a list of the schools at which training is given. 